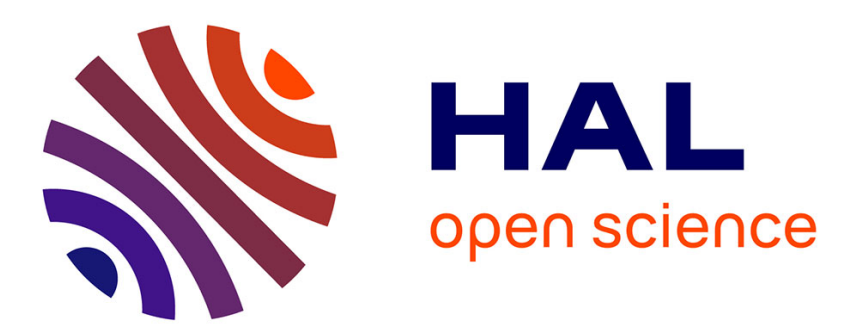

\title{
L'Argentine à la croisée des investissements énergétiques et miniers Sud-Sud
}

\author{
Marie Emilie Forget, Silvina Cecilia Carrizo
}

\section{To cite this version:}

Marie Emilie Forget, Silvina Cecilia Carrizo. L'Argentine à la croisée des investissements énergétiques et miniers Sud-Sud. Autrepart - Revue de sciences sociales au Sud, 2015, 76 (4), pp.147-159. 10.3917/autr.076.0147 . hal-03133043

\section{HAL Id: hal-03133043 \\ https://hal.science/hal-03133043}

Submitted on 22 Mar 2021

HAL is a multi-disciplinary open access archive for the deposit and dissemination of scientific research documents, whether they are published or not. The documents may come from teaching and research institutions in France or abroad, or from public or private research centers.
L'archive ouverte pluridisciplinaire HAL, est destinée au dépôt et à la diffusion de documents scientifiques de niveau recherche, publiés ou non, émanant des établissements d'enseignement et de recherche français ou étrangers, des laboratoires publics ou privés. 


\section{L'Argentine à la croisée des investissements énergétiques et miniers Sud-Sud}

\section{Marie Emilie Forget ${ }^{1}$, Silvina Cecilia Carrizo ${ }^{2}$}

Résumé À partir des années 1990, des entreprises originaires d'Amérique du Sud -Argentine, Brésil et Chili en particulier - ont multiplié et diversifié leurs investissements et leurs alliances dans la région. A la suite des réformes politico-économiques menées par certains États, les frontières de pays plus rivaux que complémentaires se sont ouvertes, générant de nouvelles opportunités pour développer les marchés régionaux. Parmi les principales entreprises internationalisées, celles exploitant les ressources naturelles sont nombreuses -énergies et minéraux principalement-. Certaines sont des entreprises publiques, des autres mixtes ou privées, d'autres encore sont des anciennes entreprises publiques privatisées. Leurs investissements s'appuient sur les institutions régionales et sur les gouvernements étatiques, qui, de leur côté, imposent leurs conditions. Dans un rapport de dépendance mutuelle, les entreprises et les États développent le secteur de l'énergie et les grandes infrastructures, facteurs clés de la cohésion socio-économique et territoriale ainsi que de la redéfinition des relations géopolitiques.

Abstract From the 1990 's, South America companies - from Argentina, Brazil and Chile in particular - have multiplied and diversified their investments and alliances in the region. Following political and economic reforms implemented by some countries, borders have opened between rival countries, generating new opportunities to develop regional markets. Among the major internationalized firms, those exploiting natural resources are many especially for exploiting minerals and energy-. Some are public companies, other mixed or private, and some are privatized from former state enterprises. Their investments are based on regional institutions and the state governments, which, in turn, impose their conditions. Due to a mutual dependence, businesses and governments develop the energy sector and large infrastructure, key factors in the socio-economic and territorial cohesion and redefine the geopolitical relations.

\section{Introduction}

D'après les Nations Unies, en 2012, pour la première fois, les pays en développement ont absorbé davantage d'investissements directs étrangers (IDE) que les pays développés ${ }^{3}$,

\footnotetext{
${ }^{1}$ Maître de conférences, Laboratoire EDYTEM Environnements, Dynamiques et Territoires de Montagne UMR 5204, Campus Savoie Technoloac, Pôle Montagne, F73376 Le Bourget du Lac cedex marie-emilie.forget@univsavoie.fr

${ }^{2}$ Chercheuse, Centro de Estudios Urbanos y Regionales. CONICET Centro de Estudios sobre el Territorio, la Energía y el Ambiente. UNNOBA. Saavedra 15 Ciudad Autónoma de Buenos Aires 1083 Argentina scarrizo@conicet.gov.ar

${ }^{3}$ Dans le rapport des Nations Unis, pour les statistiques, on considère : 1) les pays développés comme étant les membres de l'OCDE (sauf le Chili, le Mexique la République de la Corée et la Turquie) et les nouveaux membres de la Union Européenne qui ne sont pas membre de l'OCDE (Bulgarie, Chypre, Lettonie, Lituanie, Malte et Roumanie) auxquels s'ajoutent l'Andorre, les Bermudes, le Liechtenstein, Monaco et Saint Marin ; 2) les pays en transition qui regroupent les pays d'Europe du Sud-Est, les pays membres de la Communauté d'Etas indépendants et la Géorgie 3) les pays en développement : tous les autres pays [CNUCED, 2013].
} 
représentant $52 \%$ des flux d'IDE mondiaux. Les pays en développement sont aussi à l'origine de plus d'un tiers des sorties d'IED mondiales, dans la continuité d'une tendance ascendante régulière [CNUCED, 2013]. A l'intérieur de cette dynamique récente, les investissements des Suds dans les Suds augmentent. Il ne s'agit pas d'un phénomène nouveau : il existe des antécédents dans les années 1970 et 1980. Les transferts Sud-Sud peuvent en partie être expliqués par l'augmentation du niveau de développement, entendu comme le processus de réduction des écarts socio-économiques au sein des pays et entre les pays en développement et les pays développés, grâce à l'augmentation de son PIB et le réinvestissement national des dividendes générés par une croissance importante. Ainsi certains pays d'Amérique du Sud acquièrent à leur tour la capacité à investir dans des pays au développement moins avancé grâce à leurs entreprises capables de mettre en valeur leurs expériences et des savoirs plus poussés que des entreprises des pays receveurs, ce qui permet d'alimenter une phase de croissance et d'expansion.

En Amérique du Sud, à la suite des réformes néolibérales des années 1990, la tendance à l'ouverture politico-économique a amené des pays plus rivaux que complémentaires à rendre perméables leurs frontières, favorisant la constitution des marchés régionaux. Les privatisations des entreprises et des activités dans certains pays rendent ce contexte encore plus favorable aux entreprises visant le marché international. Les stratégies mises en place par les Etats dans le but d'attirer les IDE ont constamment augmenté et les pays sont entrés en compétition pour attirer des investissements et ainsi bénéficier des retombées économiques et sociales positives qui leur sont généralement associées. Alors les entreprises transnationales ainsi que les entreprises sud-américaines qui investissent de manière importante dans des pays voisins, y trouvent de nouvelles opportunités d'investissements. Dans ce processus les entreprises des pays d'Amérique du Sud ont certains avantages par rapport à des entreprises provenant d'autres régions car leurs interrelations, celles avec les Etats et les institutions régionales (Mercosur, Alliance du Pacifique, Communauté Andine des Nations par exemple) favorisent la connaissance des systèmes politiques et économiques et les liens avec leurs acteurs, rendant plus apparentes les opportunités d'investissement. Les entreprises des Suds valorisent alors une évaluation plus fine des contextes politiques et sociaux ainsi que de la qualité et de la fiabilité des infrastructures et des services. Ainsi les entreprises des Suds cherchent à exploiter les énergies et minéraux disponibles dans la région. Les entreprises publiques, mixtes, privées, parfois des anciennes entreprises publiques privatisées- et les États approfondissent une dépendance mutuelle en matière d'énergie et de grandes infrastructures, facteurs clés de la cohésion socio-économique et territoriale ainsi que de la redéfinition des relations géopolitiques.

$\mathrm{Au}$ cœur des réflexions actuelles des pays sud-américains, les investissements dans le domaine de l'énergie sont essentiels. En effet, leurs préoccupations, en termes environnementaux ou d'accès à l'énergie, rejoignent en partie celles des pays des Nords. L'enjeu est d'autant plus important que pour les plus dynamiques d'entre eux, l'Argentine, le Brésil et le Chili, s'ajoute la question de la sécurité de l'approvisionnement domestique. Les entreprises des Suds, qui possèdent une expérience majeure grâce à une trajectoire dans leur 
propre pays, savent se positionner face aux demandes de leurs voisins; y contribuent notamment la proximité législative et linguistique, la facilitation des démarches par l'existence d'accords multinationaux, l'interconnaissance et la proximité géographique. Il apparaît dans cette étude, que le processus d'investissement en énergie se fait sous la forme d'une consolidation d'acteurs anciens, mais également par la participation de nouveaux acteurs profitant d'une demande soutenue dans ce secteur. Dans le secteur minier, cette proximité et les expériences précédentes favorisent également le développement des nouvelles complémentarités.

Cet article vise à comprendre comment des entreprises des pays d' 'Amérique du Sud sont devenues moteurs dans les investissements dans d'autres pays de la région. Nous prendrons ainsi le cas d'entreprises argentines ayant investis dans des pays voisins et analyserons les investissements de ces derniers en Argentine. Pour ce faire, l'article se structure en trois parties : la première évoque les investissements en Amérique du Sud de l'entreprise pétrolière YPF -fondée par l'Etat argentin au début du XXème siècle et privatisée pendant les années 1990- ainsi que les investissements d'autres entreprises publiques sud-américaines en Argentine; la seconde montre la sud-américanisation croissante d'une entreprise pétrolière privée argentine ainsi que la présence renforcée des investissements étrangers avec une augmentation de la participation chinoise dans la région; enfin la troisième partie illustre l'émergence de nouvelles complémentarités dans le secteur minier et particulièrement dans la mise en exploitation récente de lithium.

\section{Des investissements Sud-Sud impulsés par des entreprises d'origine étatique}

Des entrepreneurs des Suds, particulièrement des entreprises publiques, commencent à investir dans des pays voisins et acquièrent de modes opératoires et organisationnels caractéristiques des investisseurs étrangers, jusqu'alors du Nord: internationalisation; rentabilité comme objectif premier; relativisation de la priorité nationale ou locale; développement d'enclaves productives avec peu de bénéfices ou d'investissements sociaux. Une sorte de transmutation des entreprises publiques vers une gestion de type transnational qui dépasse le processus d'internationalisation- a lieu.

L'histoire de l'entreprise argentine YPF Yacimientos Petrolíferos Fiscales est exemplaire de la transformation des plus grandes entreprises nationales. Fondée en 1922 par l'Etat, durant le gouvernement d'Hipólito Yrigoyen, YPF a pour objectif à sa naissance l'impulsion du développement industriel dans le respect d'une certaine indépendance économique. Son activité intégrée verticalement -depuis l'extraction de pétrole à la distribution de carburantsconditionne fortement l'organisation de cette industrie et l'activité des transnationales présentes. YPF investi à la fois dans l'exploitation des ressources des provinces de Salta, Mendoza et dans les territoires nationaux de Patagonie, dans le raffinage et la pétrochimie, dans la construction des infrastructures sociales nécessaires au développement socioéconomique des espaces directement concernés et dans la distribution dans tout le pays, pour promouvoir la présence de l'Etat et le bien-être des populations même dans les endroits les plus éloignés. L'essor d'YPF a été exemplaire pour d'autres Etats et la politique pétrolière 
nationaliste menée par l'Argentine -réussissant à limiter le pouvoir des multinationales européennes et nord-américaines naissantes- influença plusieurs pays. Le fondateur d'YPF Colonel Ingénieur Enrique Mosconi- a personnellement joué un rôle décisif, en diffusant le modèle de l'entreprise, son idéologie et l'appui à l'intégration latino-américaine, à l'occasion notamment d'un voyage provoqué par l'invitation du Président mexicain, Elías Plutarco Calles dans lequel il visite l’Uruguay (1928), le Brésil, Trinité, les Etats-Unis, le Mexique, la Colombie, le Pérou et la Bolivie ${ }^{4}$ (Larra 1957, Gadano 2006).

Tout au long du XXème siècle, YPF consolide sa forte présence au niveau national malgré les tensions avec les compagnies étrangères et le faible appui de plusieurs gouvernements nationaux. En 1990, sous le gouvernement de Carlos Menem, l'entreprise YPF -ainsi que celle de Gas del Estado- intervient pour préparer sa restructuration : forte réduction du personnel et de l'équipement, tertiarisation des activités et changement du statut de société d'Etat à celui de Société Anonyme (décret 2778 du 31 décembre 1990). Une fois restructurée -et les syndicats affaiblis-, elle est soumise à un processus de privatisation et développe une stratégie d'expansion internationale. Ces changements de caractère libéral s'inscrivent dans la Réforme de l'Etat argentin, suivant un processus global de transformation des politiques publiques auxquelles se plient, à des degrés différents, d'autres Etats sud-américains. Ainsi en 1997, au Brésil, sous la présidence de Fernando Henrique Cardoso, la législation brise le monopole du secteur pétrolier permettant l'ouverture du secteur aux capitaux étrangers. Pour la première fois depuis la fondation en 1953 de l'entreprise étatique brésilienne Petrobras, un autre groupe pétrolier entre dans l'espace brésilien : en 1998 YPF signe le premier contrat de joint-venture avec Petrobras, pour réaliser des activités d'exploration et d'exploitation offshore au Brésil.

En 1999, malgré l'expansion de l'entreprise qui prend des dimensions mondiales, l'État argentin vend les $20 \%$ d'actionnariat qui lui restaient à l'entreprise espagnole Repsol. Cette dernière rachète également $75 \%$ des actions privées. Le rachat d'YPF par Repsol permet à l'entreprise espagnole d'acquérir une position dominante sur le marché argentin -tant dans l'extraction d'hydrocarbures, que dans la production et la distribution de carburants- dans un contexte libre de concurrence. De plus, cette acquisition permet à Repsol une intégration verticale ainsi qu'un changement d'échelle dans ses activités. Au total cet achat représente pour Repsol un investissement de 15 milliards de dollars, alors que son patrimoine n'était que de 6 milliards. Cet endettement est toutefois rapidement réduit par l'augmentation des prix du pétrole et la productivité de l'entreprise achetée, ce qui lui permet d'investir en Argentine et d'autres pays d'Amérique du Sud. En juillet 2000, Repsol YPF signe un accord avec Petrobras pour obtenir une participation de $10 \%$ dans un des gisements les plus importants du Brésil : celui d'Albacora Leste, dans le bassin de Campos. Repsol devient alors un acteur fort en Amérique du Sud grâce à l'activité de production qu'YPF possédait aussi en Bolivie, en Equateur et au Venezuela et à ceux que Repsol avait en Colombie, en Equateur, au Pérou et

\footnotetext{
${ }^{4}$ La structure de l'entreprise a servi de modèle pour la constitution d'autres entreprises : ses institutions et ses infrastructures ont fait l'objet d'études, de programmes de travail et d'échanges de la part d'uruguayens et de boliviens.
} 
Trinité et Tobago (fig. 1). Elle augmente ses activités de production, diversifie les prises de risques et se positionne ainsi comme une grande compagnie à échelle mondiale ${ }^{5}$ [Carrizo, 2010 ; De Vido, Kicillof, 2012]. L'achat d'YPF fut le premier pas de Repsol dans une stratégie d'insertion et de concurrence sur le marché international, prenant comme référence des entreprises multinationales considérées compétitives et lui permet de se diversifier et de s'étendre géographiquement [De Vido, Kicillof, 2012]. Les principes de «bien commun du peuple argentin » qui régissaient YPF à ses débuts n'est dès lors plus l'objectif de l'entreprise.

Le retour des investisseurs argentins à YPF se fait par le biais du Groupe Petersen en 2007, un consortium entrepreneurial d'entreprises argentines, avant la décision, en décembre 2012, d'exproprier $51 \%$ des actions étrangères à travers une déclaration d'intérêt public national de l'exploitation et de l'exploration des hydrocarbures argentins. L'achat en février 2014 par YPF de l'entreprise pétrolière nord-américaine Apache pour plus de 800 millions de dollars vient renforcer les investissements nationaux [El Inversor energético y minero, 2013].

L'internationalisation Sud-Sud montré par le flux de capitaux argentins dans des pays de la région s'ajoute à celle d'autres entreprises sud-américaines -spécialement chiliennes et brésiliennes- qui investissent en Argentine, profitant de l'ouverture du secteur des hydrocarbures. Le cas de l'entreprise chilienne Sipetrol -filiale international de l'entreprise nationale de pétrole Enap- est exemplaire à ce propos. Parce que les gisements chiliens sont en voie d'épuisement, l'Enap s'est surtout consacrée au raffinage et aux services au Chili, en utilisant ses industries, ses ports et ses chantiers navals et elle a déployé une stratégie d'expansion internationale, pour élargir sa capacité production d'hydrocarbures hors du pays, via Sipetrol. Associée à YPF, Sipetrol exploite des plates-formes offshore dans les eaux argentines. La proximité géographique des deux pays a permis de mettre en commun des structures de gestion favorisant d'un côté, l'exploitation de gisements situés dans les marges sud du pays, peu peuplées et peu pourvues en infrastructures et de l'autre côté, la mise en valeur des installations industrielles chiliennes en terres magellanes. Stratégiquement, l'expansion de Sipetrol en Argentine l'a conduit à investir dans le transport des hydrocarbures. Les investissements de Sipetrol en Argentine ont été donc importants pour le Chili et ils ont permis de créer des infrastructures d'intégration régionale [Carrizo et Velut, 2005].

Le Brésil, autre voisin puissant, a aussi investi dans les infrastructures et l'exploitation des hydrocarbures en Argentine. Il y renforce sa politique de déploiement spatial et acquière, via la compagnie d'Etat Petrobras les entreprises argentines Petrolera San Jorge en 1999 et en 2002, la principale entreprise argentine d'origine privée du secteur des hydrocarbures, Pecom (la filière énergie de Perez Companc ${ }^{6}$ ). Elle fournit alors dans les années 2000 un cinquième du pétrole consommé en Argentine, en exporte au Chili et au Brésil et s'associe à des

\footnotetext{
${ }^{5}$ L'année de l'achat d'YPF SA, Repsol augmente ses réserves de $64 \%$, sa production d'hydrocarbures de $169 \%$, sa capacité de raffinage de $38 \%$, ses bénéfices de $59 \%$ et ses actifs de $188 \%$ (CEPAL 2001).

${ }^{6}$ Compagnie de transport maritime née en 1946, Perez Companc, transporta le matériel nécessaire à YPF pour l'installation du premier puits en Terre de Feu avant d'investir les secteurs bancaire, de la construction, de la communication, de l'agriculture, de l'exploitation du bois, du pétrole et du gaz. Elle se consolide progressivement dans ce dernier secteur, qui représente en 1991, 52\% de ses bénéfices [Saint-Lary, 2001]
} 
entreprises telles qu'Amoco, Exxon, Shell et Occidental afin d'accéder à d'autres marchés. Avec l'achat de Pecom, Petrobras augmente alors ses réserves - pour la première fois elle accède à des gisements au Venezuela- (fig.1) [Carrizo, 2010] et renforce sa capacité de raffinage particulièrement en Argentine et en Bolivie.

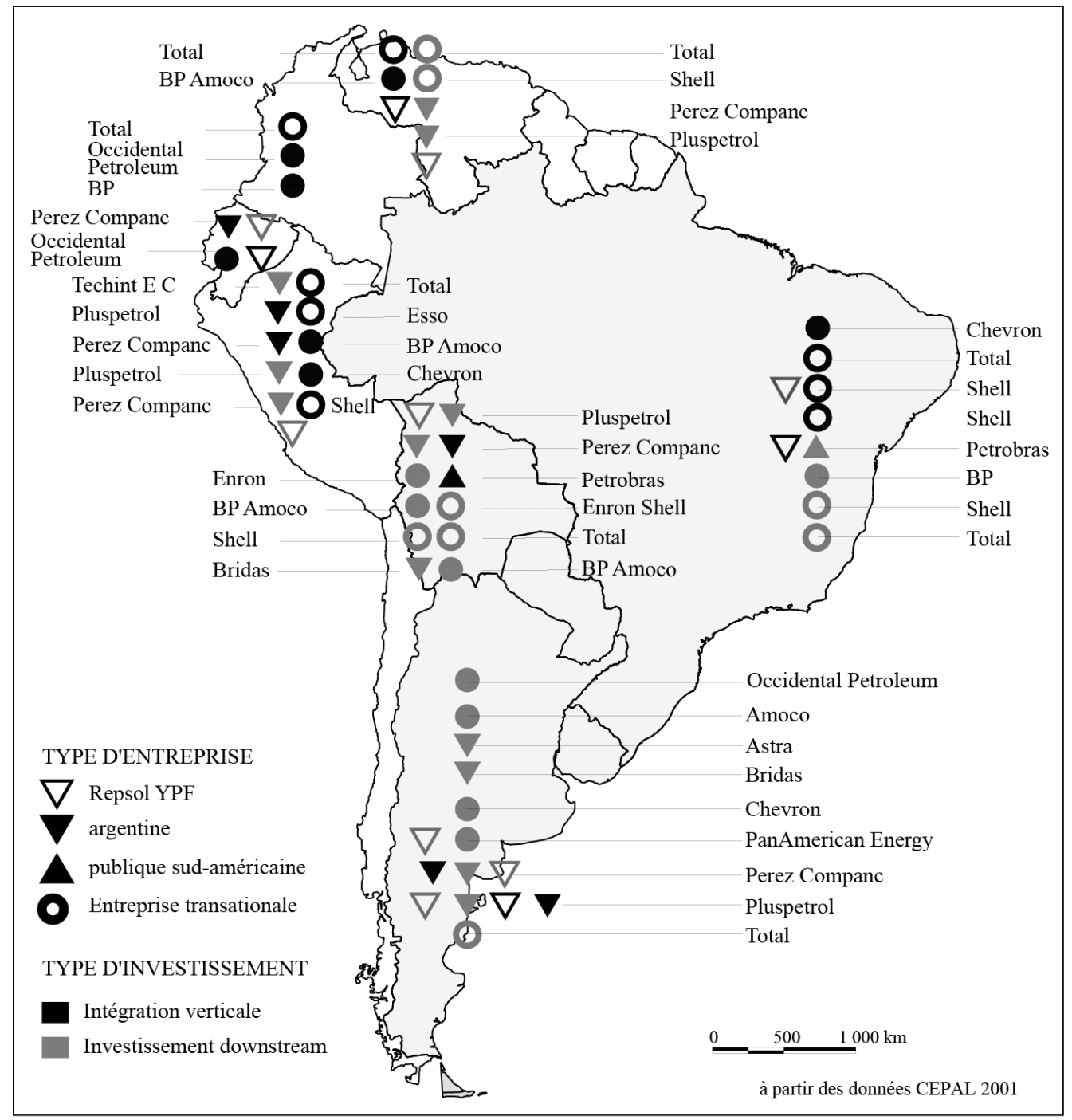

Fig. 1. Principaux investissements en Amérique du Sud des entreprises présentes en Argentine

\section{Le renforcement des investissements Sud-Sud par les acteurs privés}

Le repositionnement des Etats sud-américains dans les activités du secteur, à travers leurs propres entreprises pétrolières, s'accompagne d'un fort intérêt pour l'association avec des entreprises privées. Ce dernières sont attirées par les importantes réserves de pétrole prouvées, les deuxièmes mondiales après le Moyen Orient, représentant de l'ordre de 325 milliards de barils en 2014 [US Energy Information Administration, 2014 ${ }^{7}$ ]. C'est pourquoi les nationalisations, les contrôles économiques, l'insécurité contractuelle, les fortes subventions et l'instabilité politique qui ont eu lieu au cours du XXIe siècle n'ont pas empêché les

\footnotetext{
${ }^{7} \mathrm{http}: / /$ www.eia.gov/cfapps/ipdbproject/IEDIndex3.cfm?tid=5\&pid=57\&aid=6
} 
entreprises pétrolières transnationales de montrer un intérêt renouvelé pour la région. Les entreprises privées continuent ainsi à s'étendre au-delà des frontières de leur pays d'origine.

Le cas de la compagnie pétrolière argentine Pluspetrol est emblématique et permet d'illustrer le cas des entreprises privées d'origine sud-américaine dont l'expansion se fait dans d'autres pays de la région. Cette compagnie, fondée en 1976, a connu une trajectoire intéressante. Profitant d'un savoir-faire propre aux pays avec du pétrole, l'entreprise a largement investi au-delà des frontières argentines. En effet, après avoir mis en exploitation l'usine Yacimiento Centenario en 1977, l'entreprise s'est rapidement tournée vers des territoires situés dans les pays du Sud. Les premiers investissements se font dans des pays sud-américains avec notamment les premières explorations en Colombie en 1977 et la première exploitation bolivienne dans les années 1990. Puis l'entreprise a trouvé l'opportunité de se développer dans les pays d'Afrique du Nord, comme l'Algérie en 1993 et la Tunisie en 1994. Avec une production en 2013 de plus de 415 millions de barils équivalent pétrole, les investissements de cette société s'orientent préférentiellement vers les pays proches comme la Bolivie et le Pérou -où elle développe le méga-gisement gazier Camisea-, concurrençant d'autres entreprises de grande ampleur comme Petrobras (Brésil) ou l'ex-Repsol YPF redevenue YPF à majorité argentine. A l'image des plus grandes entreprises transnationales, Pluspetrol s'est également positionnée sur le marché de l'exploration concentrant ses recherches au Venezuela et en Colombie mais également en Angola.

Les grandes entreprises pétrolières transnationales sont présentes sur le continent depuis le début du XXe siècle et en Argentine, la présence des plus grandes a été permanente. Aujourd'hui il existe un intérêt particulier car elles investissent dans l'exploration et la production de ressources non conventionnelles, dont les coûts et les technologies sont difficilement accessibles aux entreprises nationales. De ce fait en 2013, sous le gouvernement de la Présidente argentine Cristina Fernandez de Kirchner, YPF a signé un accord avec la firme Chevron pour développer le gisement d'hydrocarbures non conventionnels de Vaca Muerta dans la province de Neuquén et en 2014 le Congrès a adopté une nouvelle loi sur les hydrocarbures qui apporte des avantages et des facilités pour ce type d'investissements.

L'intérêt de la Chine, cinquième émetteur mondial d'IDE, semble particulièrement fort en Amérique du Sud [Wu, 2007]. Leurs liens commerciaux se resserrent depuis les années 1990, ces derniers lui fournissant principalement du soja, des minéraux et du pétrole [Cepal, 2013] ; mais depuis la deuxième décennie du XXIe siècle, elle y investit de plus en plus pour répondre à ses besoins en matières premières, étant le plus grand consommateur mondial d'électricité et le deuxième de pétrole [Slipak, 2014 ; Bekerman, Dulcich, Moncaut, 2014]. Ainsi, un accord entre les états chinois et argentin, daté de 2010, sur l'énergie stipule que dans les dix ans à venir la Chine injectera 5 milliards de dollars dans la prospection du pétrole et du gaz en Argentine [Arès, 2011]. Qui plus est, pendant la visite du président Hu Jintao au Brésil, onze accords ont été signés avec le gouvernement brésilien de Lula da Silva en 2009 validant l'investissement par la Chine de 1 milliard de dollars, sur les années à venir dans les secteurs du transport et de l'énergie [Wu, 2007]. Le fait que la Chine ait approfondi et élargi ses relations avec les pays d'Amérique du Sud, en particulier avec le Venezuela a attiré 
l'attention et provoqué la méfiance et l'inquiétude des Etats-Unis dont une partie de l'approvisionnement en pétrole provient de cette région du monde. L'influence croissante de la Chine suscite ainsi des tensions. L'intensification des relations entre la Chine et le Venezuela est perçue par certains hommes politiques et spécialistes américains comme une intrusion dans l'arrière-cour de l'Amérique, et même comme le début d'une sinisation de l'Amérique latine qui génère certaines tensions géopolitiques [Johnson, 2005].

Aujourd'hui on assiste toutefois à l'émergence de processus de coopération entre pays sudaméricains se basant sur une complémentarité entre les ressources financières, les savoir-faire et les infrastructures de chaque pays, pour exploiter les ressources naturelles.

\section{Opportunités de complémentarités ouvertes dans le secteur minier}

Dans le secteur minier, l'Amérique du Sud reste un réservoir à développer. Du fait d'un manque d'infrastructures dans certaines régions périphériques ou d'entreprises capables d'exploiter certaines ressources, il existe un intérêt à attirer des investissements directs étrangers. Bien qu'il existe un savoir-faire dans de nombreux domaines énergétiques comme ceux présentés plus haut, il manque parfois certains maillons dans la chaîne de production, qui peuvent être proposés par des entreprises des pays voisins : c'est le cas des compagnies chiliennes pour l'exploitation du lithium ou du cuivre dans la Cordillère andine.

Le lithium est un minéral, dont les réserves sud-américaines se trouvent réparties dans un «Triangle » entre l'Argentine, la Bolivie et le Chili, qui représentent $70 \%$ des réserves mondiales. Son exploitation à petite échelle ${ }^{8}$ est en projet depuis quelques années. Des entreprises chiliennes se positionnent sur le marché argentin en faisant valoir leur savoir-faire pour rendre disponible cette ressource. Le Chili est en effet le premier producteur mondial de lithium avec $40 \%$ de la production grâce à l'exploitation du Salar d'Atacama. L'Argentine est le $4^{\text {ème }}$ producteur mondial mais possède un important potentiel de développement ${ }^{9}$. Des entreprises chiliennes ont commencé à développer l'exploitation de ce minéral dans leur propre pays où elles ont acquis l'expérience et la connaissance. Récemment elles ont obtenu des concessions d'exploitation dans les provinces de Salta, Jujuy et Catamarca. En effet, les conditions d'exploitation sont très contraignantes puisqu'elles demandent une gestion d'un groupe de travailleurs à haute altitude dans des sites isolés (à titre d'exemple, le Salar del Hombre Muerto est situé à plus de 4000 d'altitude dans un climat aride, sachant que les travailleurs sur ces chantiers ne sont pas forcément originaires de la Cordillère et que les protocoles d'exploitation ne sont pas nécessairement établis pour ces altitudes). Ces entreprises chiliennes cherchent également à se positionner dans ce secteur en Bolivie.

\footnotetext{
${ }^{8}$ On se réfère ici à une petite échelle géographique c'est-à-dire de grande ampleur et intéressant de larges aires géographiques.

${ }^{9}$ Dans les provinces de Catamarca, Salta et Jujuy comptent de l'ordre de 400000 hectares de salars qui sont autant de gisements potentiels.
} 


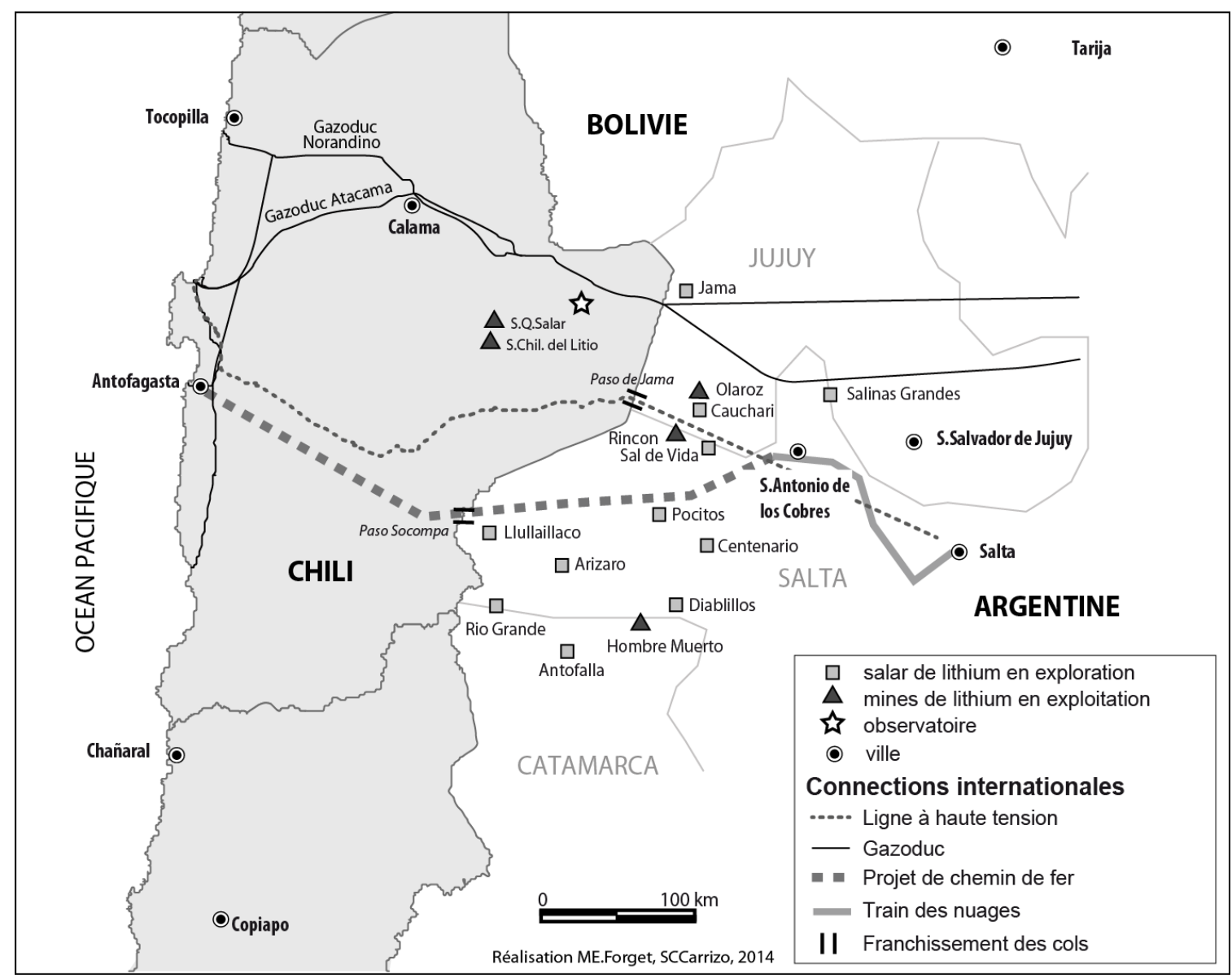

Fig.2 Exploitations de lithium dans les salars ${ }^{10} \mathrm{du}$ Nord-ouest argentin

En Argentine les ressources minières comme les hydrocarbures sont du domaine des Provinces, habilitées à donner les lots d'exploitation en concession. Dans ce contexte, le gouverneur de la Province de Salta, Juan Manuel Urtubey, a initié le rapprochement entre le Chili et l'Argentine pour l'exploitation du lithium en invitant officiellement les représentants de la société chilienne Sociedad Química y Minera (SQM) à explorer les salars. Il convient de noter que l'entreprise SQM est le plus grand producteur mondial de ce minéral grâce à l'exploitation de gisements localisés dans le nord du Chili, à proximité d'Antofagasta (fig 2). Avec 12000 employés et des bénéfices s'élevant à plus de 2 milliards de dollars annuels, l'entreprise exporte principalement vers la Corée du Sud et la Chine. D'autres entreprises chiliennes se sont positionnées, proposant des offres d'investissement à la Province de Salta. Parmi elles, le groupe Lucksic propose d'exploiter les gisements de Sal de Vida et le Salar del Hombre Muerto (fig 2), avec des investissements supérieurs à 200 millions de dollars dans chacun des deux sites. Ces investissements sont cependant soumis à conditions. En effet, l'entreprise n'envisage de concrétiser ces opérations que si la Province parvient à garantir la réactivation du chemin de fer unissant Antofagasta (Chili) à Salta (Argentine) et s'engage à améliorer l'état des routes d'accès pour faciliter l'exportation du minéral vers les marchés

\footnotetext{
${ }^{10}$ Un salar est un désert de sel. Il s'agit d'un lac plus ou moins temporaire aux rives changeantes dont les sédiments sont essentiellement constitués par des sels et dans lesquels se trouvent des concentrations importantes en lithium.
} 
asiatiques. La voie d'accès et de sortie se fait par le Nord du salar au moyen de la route provinciale $\mathrm{N}^{\circ} 17$ dans la Province de Salta (qui prolonge au Nord la route provinciale $\mathrm{N}^{\circ} 43$ reliant le salar à la ville d'Antofagasta de la Sierra). Par cette route de $100 \mathrm{~km}$, les sels de lithium seraient acheminés vers le chemin de fer reliant San Antonio de los Cobres au col frontière du Paso Socompa (fig 2). Cette voie ferrée est la prolongation vers l'ouest du « train des nuages » et continue au Chili vers le port d'Antofagasta. L'intérêt à attirer les capitaux s'est par ailleurs concrétisé par la signature d'une convention avec l'entreprise Ferronor en charge de déterminer les travaux nécessaires à la remise en état du chemin de fer, aujourd'hui inactive $^{11}$. De cette façon l'augmentation des échanges, des investissements et la mise en connexion d'infrastructures fait place à une régionalisation dans ce «Triangle du lithium » entre pays d'Amérique du Sud. Cette régionalisation bénéficient alors d'une proximité géographique, de liens culturels, historiques et linguistiques; de la connaissance des systèmes politiques et des politiques commerciales et qui de plus promeuvent de potentiels accords commerciaux et/ou institutionnels.

Les négociations à l'œuvre autour d'une exploitation chilienne des salars du Nord argentin, permettraient de renforcer le positionnement des pays de la région dans les secteurs stratégiques comme le lithium, qui sont aujourd'hui détenus dans leur grande majorité par des sociétés des Nords comme le présente le tableau ci-dessous.

\begin{tabular}{|c|c|c|c|c|c|c|}
\hline Nom du gisement & Province & $\begin{array}{l}\text { Entreprise } \\
\text { exploitante }\end{array}$ & Nationalité & Superficie & $\begin{array}{l}\text { Superficie } \\
\text { octroyée }\end{array}$ & $\begin{array}{l}\text { Altitude } \\
\text { moyenne }\end{array}$ \\
\hline Salar del Rincón & Salta & Ady Resources & Australie & 420 & 363 & 3660 \\
\hline Salar de Olaroz & Jujuy & Orocobre/ Toyota & Australie & 160 & 230 & 3800 \\
\hline Salar de Olaroz & Jujuy & $\begin{array}{l}\text { Minera Exar } / \\
\text { Mitsubishi }\end{array}$ & Canada & 160 & & 3800 \\
\hline $\begin{array}{l}\text { Salar del Hombre } \\
\text { Muerto }\end{array}$ & $\begin{array}{l}\text { Catamarca / } \\
\text { Salta }\end{array}$ & Lithium One & Canada & 588 & 133 & 3750 \\
\hline $\begin{array}{l}\text { Salar del Hombre } \\
\text { Muerto }\end{array}$ & $\begin{array}{l}\text { Catamarca / } \\
\text { Salta }\end{array}$ & FMC Lithium Corp & Etats-Unis & 588 & 300 & 3750 \\
\hline Salar de Diablillos & Salta & Rodinia Lithi & Canada & 40 & 56 & 4000 \\
\hline $\begin{array}{l}\text { Salares Incahuasi, } \\
\text { Pocitos y Arizano }\end{array}$ & Salta & $\begin{array}{l}\text { Minera Exar } / \\
\text { Mitsubishi }\end{array}$ & Canada & 2135 & 2687 & 3750 \\
\hline Salar Centenario & Salta & $\begin{array}{ll}\begin{array}{l}\text { Bolera } \\
\text { (Bolloré) }\end{array} & \text { Minera } \\
\end{array}$ & France & 85 & 67 & 3760 \\
\hline Salar Cauchari & Jujuy & $\begin{array}{l}\text { Lithium Américas } \\
\text { Corporation }\end{array}$ & Canada & 350 & 436 & 3850 \\
\hline Salinas Grandes & Salta / Jujuy & $\begin{array}{l}\text { South American } \\
\text { Salars (Orocobre) }\end{array}$ & Australie & 220 & 385 & 3520 \\
\hline Salar de Pozuelos & Salta & Ekeko & $\begin{array}{l}\text { Corée du } \\
\text { Sud }\end{array}$ & 80 & 110 & 3710 \\
\hline Salar de Llullaillaco & Salta & $\begin{array}{lr}\text { Minera } & \text { Solitario } \\
\text { Argentina } & \text { (TNR } \\
\text { Gold) } & \\
\end{array}$ & Canada & 130 & 154 & 3750 \\
\hline
\end{tabular}

Tab.1. L'exploitation de lithium dans les provinces argentines est principalement faite par des entreprises des pays des Nords [El Inversor energético y minero, 2013].

En Argentine, les investissements miniers, qui jusqu'en 2005 ne figuraient pas comme un secteur préférentiel, constituent aujourd'hui le principal secteur d'investissement, avec une croissance de $91 \%$ en 2011 [Banco Central de la República Argentina, 2011]. Parmi eux les

\footnotetext{
${ }^{11} \mathrm{http} / / / \mathrm{www}$. iprofesional.com
} 
investissements chiliens augmentent rapidement depuis 2010, avec une croissance de $22 \%$ enregistrée en 2011.

\section{Conclusion}

Les investissements Sud-Sud au sein de l'Amérique du Sud ont parfois été impulsés par des entreprises étatiques, particulièrement par les grandes entreprises nationales en charge de l'exploitation des hydrocarbures, cherchant de nouvelles opportunités dans les pays voisins dont ils connaissent mieux les contextes politico-économiques. En définissant la rentabilité comme objectif principal en s'internationalisant, le développement national est passé au second plan. De plus, elles concurrencent progressivement les entreprises des pays voisins en reproduisant les principes de fonctionnement des entreprises transnationales et en acquérant un mode d'organisation qui se rapproche de celui traditionnellement attribué aux entreprises des Nords. Cela s'expliquerait, d'une part, par leur mise en concurrence sur les marchés internationaux les incitant à intégrer des pratiques propres aux grandes multinationales et, d'autre part, par des modèles de fonctionnement ayant parfois fait intervenir au cours de leur développement un certain nombre d'acteurs financiers des Nords. La dépendance mutuelle qui s'établit dans le domaine de l'énergie -surtout dans le secteur des hydrocarbures- entre Etas et entreprises joue également un rôle important.

L'Amérique du Sud en général et l'Argentine en particulier, connaissent depuis longtemps des tensions sur le marché de l'énergie dont le développement a donc été soutenu par les différents gouvernements, via d'entreprises nationales ou privées. Les questions de sécurité et d'indépendance énergétiques sont d'ailleurs toujours des enjeux d'actualité. Depuis le début du XXIe siècle, la question de la valorisation des ressources naturelles est également devenue centrale, notamment en ce qui concerne les ressources minières. Les entreprises privées sudaméricaines, argentines, chiliennes et brésiliennes, cherchent à exporter dans d'autres pays de la région un savoir-faire développé, notamment dans les domaines énergétique et minier. Pour cela, elles s'appuient sur des relations diplomatiques privilégiées avec les Etats voisins et se fondent sur une très bonne connaissance des environnements et des contextes socio-politicoéconomiques.

La concurrence entre les différents acteurs économiques peut toutefois prendre sur le territoire des formes de complémentarité, notamment par la disponibilité différenciée d'infrastructures et des ressources naturelles, comme c'est le cas en terres magellanes. Les investissements prennent alors souvent la forme de partenariat public-privé ou sont négociés entre grandes firmes privées. La stratégie de diversification des ouvrages et l'extension géographique semble alors faire partie de la stratégie de nombreux acteurs qui voient dans les pays voisins une manière d'étendre leurs opportunités, leurs sources de production ou leurs marchés. Il se dessine alors une géographie de l'investissement qui met en avant l'expertise acquise au cours des périodes antérieures, grâce à l'interconnaissance et aux liens transfrontaliers dont profitent les entreprises des pays sud-américaines. Ces investissements Sud-Sud, qui génèrent de l'activité productive dans le sous-continent, renforcent la mise en place de structures d'échanges multinationales et l'interdépendance des économies, et font ainsi avancer une intégration régionale. 


\section{Bibliographie}

AMILHAT SZARY AL. [2010], « Les entreprises minières dans la gouvernance territoriale Entre négociations de pouvoir et rivalités territoriales, qui porte le développement aux habitants ? », Revue Géographique de l'Est, vol. 50 / 3-4 : http:// rge.revues.org/3132| (page consultée le 16 janvier 2014)

ARes M. ET AL. [2011], «La Chine et l'Amérique latine : le grand chambardement ? », Revue Tiers Monde, 2011/4 n²08, p. 65-82.

Banco Central de la República Argentina [2011], Las inversiones directas en empresas residentes a fines del 2011, juillet 2011, 29 p.: http:// www.brca.gov.ar/pdfs/estadisticas/inversiones\%20directas\%2031\%2012\%2011.pdf $\quad$ (page consultée le 13 mars 2014)

BeKerman M., Dulcich F., Moncaut N. [2014], "La emergencia de China y su impacto en las relaciones comerciales entre Argentina y Brasil" Problemas del Desarrollo. Revista Latinoamericana de Economía vol. 45, núm. 176, enero-marzo, 2014, pp. 55-82 Universidad Nacional Autónoma de México, Distrito Federal de México

Bianco C., Moldovan P., Porta F. [2008], La internacionalización de las empresas brasileñas en Argentina, CEPAL, Publication des Nations Unies, Santigo du Chili, 74p.

CARrizo S., Velut S. [2005] «Nouvelles territorialités en Amérique australe. Activités énergétiques et intégration dans les terres et les mers magellanes », L'Espace géographique, 2005/2 tome 34 , p. $161-175$

CARRIZO, S. C. 2010.Hydrocarbures en Argentine: réseaux, territoires, intégration. Éditions universitaires européennes. Sarrebruck. 398 p.

CePAl [2007], Promoción del comercio y la inversión con China, Santiago de Chile, noviembre

CEPAL [2001] La inversión extranjera en América Latina y el Caribe. CEPAL. Santiago de Chile.

DE Vido J., KicILlof A. [2012], YPF El informe Mosconi Ministerio de Planificación Federal, Inversión pública y Servicios. Ministerio de Economía y Finanzas públicas, Buenos Aires $91 \mathrm{p}$.

Johnson C. [2005] « The real "China Threat" », Asia Times, 21 mars 2005: http://www.atimes.com/atimes/China/GC19Ad05.html (page consultée le 18 mars 2014)

EL COMERCIO [2013], “Argentina protesta por acuerdo firmado entre YPF y Chevron”, El Comercio, 16 juillet 2013: http://elcomercio.pe/mundo/actualidad/argentina-protesta-acuerdofirmado-entre-ypf-chevron-noticia-1604734, (page consultée le 17 mars 2014)

EL INVERSOR ENERGÉTICO Y MINERO, [2013] "Litio y potasio, las grandes apuestas mineras del Norte argentino" 2013, año 5 N56, mai 2013, Editorial PGQ, Buenos Aires 
GADANO N. [2006], Historia del petróleo en la Argentina. 1907-1955: Desde los inicios hasta la caída de Perón. Edhasa, Buenos Aires

LARRA R. [1957], Mosconi, General del Petróleo. Futuro, Buenos Aires

Nicolo Gligo S. [2007], Políticas activas para atraer inversión extranjera directa en América Latina y el Caribe, Red de Inversiones y Estrategias Empresariales Unidad de Inversiones y Estrategias Empresariales, División de Desarrollo Productivo y Empresarial, SERIE Desarrollo productivo, Santiago de Chile, $\mathrm{n}^{\circ} 175,131 \mathrm{p}$.

ORgANiSATION DES NATIONS UNIES [2013], Rapport sur l'investissement dans le monde. Les chaînes de valeur mondiales : l'investissement et le commerce au service du développement, CNUCED, 53 p. : http://unctad.org/fr/PublicationsLibrary/wir2013overview_fr.pdf (page consultée le 13 mars 2014)

SAINT-LARY, B. [2001], "La actividad hidrocarburífera: el marco necesario para entender las realidades locales" in COLANTUONO, SAINT-LARY B. ET ALLI, [2001], "Petróleo y desarrollo local. Centros norpatagónicos en busca de alternativas Universidad Nacional del Comahue". Departamento de Geografía. Neuquén.

SLIPAK A. [2014], "América Latina y China: ¿cooperación Sur-Sur o «Consenso de Beijing»?" Revista Nueva sociedad No 250, marzo-abril

Soria, C. [2005], Camisea: por qué cuesta tanto el gas barato?, Facultad Latinoamericana de Ciencias Sociales, Sede Académica de Ecuador, 118 p.

Wu L. [2007], « La quête chinoise d'énergie en Amérique latine et ses implications géopolitiques », Outre-Terre, 2007/1 n 18, p. 393-405 